\title{
Neuroprotective and Antioxidant Activities of Fraction Isolated from Methanolic Extract of Sapindus laurifolia on Scopolamine Induced Dementia
}

\author{
Nagaraju Bandaru, A. Ramu', S. Vidhyadhara' \\ Departments of Pharmacology and 'Pharmaceutics, Chebrolu Hanumaiah Institute of Pharmaceutical Sciences, Guntur, Andhra Pradesh, India
}

\begin{abstract}
Background: Phytotherapy is considered a complementary approach for preventing and treating many diseases. Plants are rich source of bioactive secondary metabolites of a wide variety such as saponins, tannins, terpenoids, alkaloids, and flavonoids. Sapindus laurifolia (fam: Sapindaceae), well known as soapnuts, are used medicinally as an expectorant, emetic, contraceptive, and for treatment of excessive salivation, epilepsy, chlorosis, and migraines. Objective: To isolation of fractions, estimation of total flavonoid content, in vitro antioxidant and in vivo neuroprotective activity of S. laurifolia fruit kernel. Materials and Methods: In this study, in vitro antioxidant activity is estimated by 2,2-diphenyl-1-picrylhydrazyl assay method. Neuroprotective activity is estimated by scopolamine-induced dementia method. In neuroprotective method, behavioral parameters were checked by escape latency (EL) time (s) by using the Morris water maze, active avoidance testing using elevated plus maze, and neurochemicals such as glutathione, catalase (CAT), superoxide dismutase (SOD), and Cholinesterase were estimated. Results: In scopolamine-induced dementia, increased EL time, active avoidance and neurochemicals such as glutathione, CAT, SOD and cholinesterase decreased. Whereas standard and Fraction S-treated animals decreased in EL time, active avoidance and increased locomotor activity, neurochemicals such as glutathione, CAT, SOD, and cholinesterase also increased. Conclusion: The present study clearly demonstrates that methanolic extract of $S$. laurifolia Fraction A (50 and $100 \mathrm{mg} / \mathrm{kg}$ ) kernel significantly attenuate scopolamine-induced dementia by improving the learning, memory, antioxidant potentiality, and anti-acetyl cholinesterase activity. Therefore, this Fraction A can be a potential novel therapeutic strategy for controlling neurodegenerative dementia especially Alzheimer's disease. Yet, advance studies are needed to characterize the active compound(s) and expose the possible mechanism of action.
\end{abstract}

Key words: Antioxidant, dementia, Isolation, Neuroprotective, sapindus

\section{SUMMARY}

- In this research MESL and MESL fraction A significantly shows neuroprotective activity in scopolamine induced dementia by decrease escape latency time and increases antioxidants levels in brain, so this antioxidants prevents the formation of oxidative stress and decreases progression of dementia. So
Sapindus laurifolia became one of the major drug treatment for dementia in future.

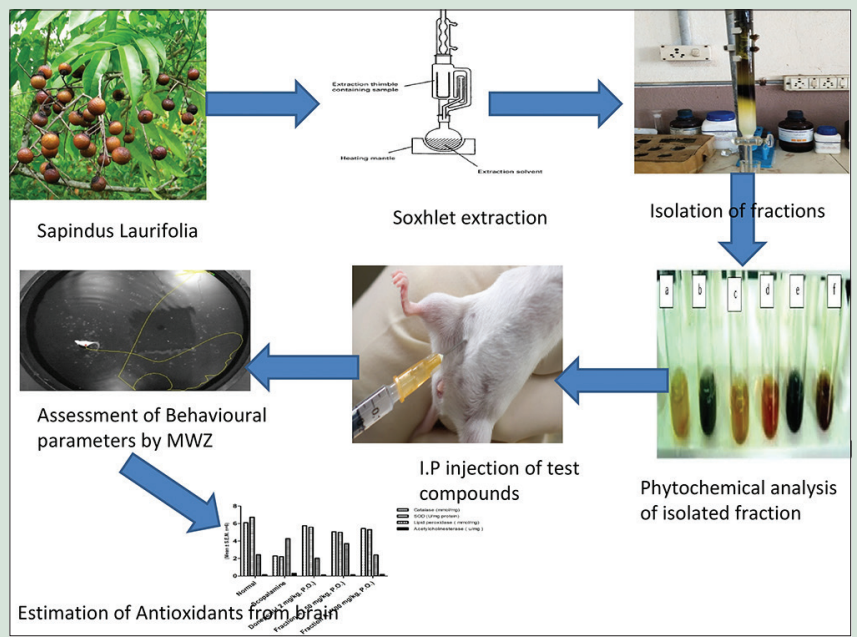

Abbreviations Used: AD: Alzimer's disease; NFTs: Neurofibrillary tangles; Ach: Acetylcholine; AchE: Acetylcholinesterase; CAT: Catalase; GSG-Px: Glutathione peroxidases; GSR: Glutathione reductase; GSH: Glutathione; GST: Glutathione transferase; WHO: World Health Organisation; DPPH: 2,2-diphenyl-1picrylhydrazyl; MESL: Methanolic extract of Sapindus laurifolia.

Correspondence:

Mr. Nagaraju Bandaru,

Department of Pharmacology, Chebrolu

Hanumaiah Institute of Pharmaceutical Sciences, Chowdavaram. Guntur, Andhra Pradesh, India. E-mail: bnrajupharma@gmail.com DOI: 10.4103/pr.pr_50_19
Access this article online Website: www.phcogres.com Quick Response Code:

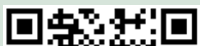

\section{INTRODUCTION}

Cognition is a process of storing an arrangements of facts in a regular method by which one becomes attentive of their surroundings, objects, and views ${ }^{[1]}$ Impairment of cognition is one of the foremost health complications into normal aged life along within some neurological disease conditions, including Alzheimer's disease (AD). ${ }^{[2]} \mathrm{AD}$ is an age-related progressive neurodegenerative disorder which is associated with the unavoidable loss of cognitive function. This is the most common form of dementia, accounting for approximately $60 \%$ of all cases of impairing memory. ${ }^{[3]}$ Globally, at present, 30 million people are affected by this disease and it is estimated that by 2050 more than 115 million people will have dementia. ${ }^{[4,5]}$ This disease is characterized by deposition of senile plaques, amyloid- $\beta$ (A $\beta)$ and development of neurofibrillary tangles (NFTs) in the cerebral cortex

and subcortical gray matter. ${ }^{[6]}$ Alzheimer's patient's brain has elevated levels of acetylcholinesterase (AChE), which is responsible for the breakdown of acetylcholine (ACh). ACh is a neurotransmitter which plays an important role for as proper functioning of as the central

This is an open access journal, and articles are distributed under the terms of the Creative Commons Attribution-NonCommercial-ShareAlike 4.0 License, which allows others to remix, tweak, and build upon the work non-commercially, as long as appropriate credit is given and the new creations are licensed under the identical terms.

For reprints contact: reprints@medknow.com

Cite this article as: Bandaru N, Ramu A, Vidhyadhara S. Neuroprotective and antioxidant activities of fraction isolated from methanolic extract of Sapindus laurifolia on scopolamine induced dementia. Phcog Res 2020;12:119-26. 
cholinergic system. ${ }^{[7]}$ There is no treatment for AD. However, some AChE inhibitors such as physostigmine, tacrine, and donepezil may help keep symptoms from getting worse for a limited time. Therefore, it is lucrative to explore the usefulness of medicinal plants for the treatment of various cognitive disorders. Scopolamine is commonly injected i.p route to study cognitive deficits in experimental animals. It is structurally similar to the neurotransmitter Ach and exerts its function by blocking the muscarinic Ach receptors that lead to cholinergic dysfunction and impaired cognition. ${ }^{[8]}$ In recent times, studies result that impairment of memory in the scopolamine-induced animal model is linked with increased oxidative stress within the brain due to an alteration of brain antioxidant enzymes. ${ }^{[9-11]}$ Increasing evidence highlights that free radical-mediated oxidative stress is responsible for AD. ${ }^{[12]} \mathrm{In}$ order to leads to a normal life, oxidation is necessary for biological reactions or processes that lead to the generation of reactive oxygen species (ROS) as byproducts. Proper functioning of as the human body depends on the redox homeostasis i.e., the balance between oxidation and antioxidation. ${ }^{[13]}$ In case of late-onset sporadic $\mathrm{AD}$, imbalances between oxidation and antioxidant factors induce cellular and molecular abnormalities. ${ }^{[14]}$ The neuronal network i.e., the brain is more prone to the oxidative damage because of a high level of long-chain polyunsaturated lipids in neuronal cell membranes, high oxygen consumption, high metabolic rate of transitional metals and poor antioxidative defense. ${ }^{[15,16]}$ Several studies suggested that after oxidative stress, senile plaques and NFTs are formed in $\mathrm{AD} .^{[17]}$ In addition to this, the antioxidant defenses of the brain are modest. The content of catalase (CAT), glutathione peroxidase (GSH-Px) and Vitamin E in the brain is less as compared to the liver ${ }^{[18]}$ Antioxidants are exogenous or endogenous substances that prevent oxidation and act against oxidative stress associated with deleterious effects on the cellular system. ${ }^{[19]}$ Antioxidants are also associated in with scavenging free radicals, preventing free radicals chain reactions, and improve antioxidant status in patients. ${ }^{[20]}$ In order to prevent or slow the progression of free radicals mediated oxidative stress, brain antioxidant defense enzymes such as CAT, GSH-Px, GSR, reduced glutathione (GSH), and glutathione-S-transferase (GST) play an important role. ${ }^{[21]}$ In recent years, there has been increased interest in the therapeutic use of antioxidants in the treatment of disease associated with free radicals mediated oxidative stress. As said by the World Health Organization more than $80 \%$ of the world's population trusts on complementary and alternative medicine for their medical care. ${ }^{[22]}$ The use of medicinal plant and plant products is increasing day by day. About 3.5 billion people in developing countries, primarily depended on with medicinal plants and herbal medicine around for their health-care needs. ${ }^{[23]}$ On the other hand, in Western society, the rejection of synthetic or biomedical products has become a growing trend and allowed for a rise in the demand for natural medicines. ${ }^{[24]}$ In the treatment of cognitive dysfunctions, the phytoconstituents of medicinal plants play a central part. In the treatment of AD medicinal plants such as Ginkgo biloba, Bacopa monnieri, and Huperzia serrata have been widely examined. ${ }^{[25-27]}$

Sapindus laurifolia (fam: Sapindaceae), well known as soapnuts, is used medicinally as an expectorant, emetic, contraceptive, and for treatment of excessive salivation, epilepsy, chlorosis, and migraine. S. laurifolia is a popular ingredient in ayurvedic shampoos and cleansers. They are used in ayurvedic medicine for the treatment of eczema, psoriasis, and for removing freckles. Soap nuts have gentle insecticidal properties and are traditionally used for removing lice from the scalp.

In the current research, the main objective is to the isolation of fractions, estimation of total flavonoid content, in vitro antioxidant and in vivo neuroprotective activity of $S$. laurifolia fruit kernel.

\section{MATERIALS AND METHODS}

\section{Animals for the experiment}

Healthy adult albino Wistar rats weighing 200-250 g of Wistar rats were selected for the study. Animals were housed in appropriate uniform hygienic conditions and fed with standard pellet diet (Amrul Laboratory ANIMAL Diet) and water ad libitum. Animal studies had the approval of IACE by the committee for the purpose of control and supervision of experiments on animals.

\section{Plant collection \\ Authentication of plant}

S. laurifolia is authenticated by the Department of Botany, AKRG Degree College, Bhimavaram, Andhra Pradesh, India.

S. laurifolia fruits are collected from the local market of Bhimavaram, West Godavari district, Andhra Pradesh, and authenticated by Dr. P. Prasanna Kumari Department of Botany, D. N. R College, Bhimavaram A voucher specimen (3015) was kept at the Department of Pharmacology, Shri Vishnu College of Pharmacy, Bhimavaram, Andhra Pradesh.

\section{Preparation of plant extract Extraction}

Coarsely powdered (500 g) were successively extracted with petroleum ether for 7 days to remove fatty matter. The defatted marc was then subjected to soxhlet extraction with $95 \%$ ethanol to obtain methanolic extract. The methanolic extract was evaporated under reduced pressure at low temperature $\left(300^{\circ} \mathrm{C}\right)$ to dryness and brownish yellow color extracts was obtained..$^{[28]}$

\section{The isolation of fractions from methanolic extract using column chromatography}

The glass column containing stationary phases (silica gel) is encountered slowly from the top with a liquid solvent (mobile phase) that flows down the column with the help of external pressure applied. This technique is used for the purification of compounds from a mixture. Once the column is ready, the sample is loaded with the top of the column. The mobile phases are then allowed to flow down through the entire column. Components in mixtures have a different ability to interact with stationary phases (silica gel) and the mobile phase, there by flowing along the mobile phase at different time intervals. In this way, the separation of compounds from the mixture is achieved. The individual compounds are collected as fractions and analyzed further for structure elucidation [Table 1].

\section{Preliminary phytochemical screening}

The preliminary phytochemical investigations were carried out for the qualitative determination of phytochemicals.

\section{Acute toxicity studies}

Acute toxicity studies were performed according to the organization for economic co-operation and development OECD guidelines 423 (2001).

Table 1: Gradient solvent system used in column chromatography

Gradient solvent system used in column chromatography for the isolation of fractions in Sapindus laurifolia

\begin{tabular}{lcc}
\hline Solvent system & Ratio & Fraction \\
\hline H (\%) & 100 & - \\
H: EA & $95: 5$ & A \\
H: EA & $70: 30$ & B \\
H: EA & $50: 50$ & C \\
Only EA (\%) & 100 & D \\
Only M (\%) & 100 & E \\
\hline
\end{tabular}

H: Hexane; EA: Ethyl acetate; M: Methanol 
Animals were divided into four groups $(n=4)$ and fasted for $4 \mathrm{~h}$ with free access to water only. The methanolic extract of S. laurifolia (MESL) Fraction A was administered orally in doses of 550,300 and $2000 \mathrm{mgkg}$ to different groups of mice and observed for physical/behavioral and mortality changes at $30 \mathrm{~min}, 5,25,58 \mathrm{~h}$, and 1 week.

\section{Free radical scavenging activity (2,2-diphenyl-1-picrylhydrazyl)}

2,2-diphenyl-1-picrylhydrazyl (DPPH) is one of the widely used for testing preliminary radical scavenging activity of the plant fraction A. Scavenging of DPPH radical is related to the inhibition of lipid peroxidation (LPO). DPPH is usually used as a substance to evaluate the antioxidant activity. Antioxidants either transfer an electron or a hydrogen atom to DPPH thus neutralizing its free radical character. $\mathrm{DPPH}$, which is based on the ability of DPPH a stable free radical, to decolorize in the presence of antioxidants, is a direct and reliable method for determining radical scavenging action. The DPPH assay has been largely used as a quick, reliable and reproducible parameter to search the in vitro general antioxidant activity of pure compounds as well as plant extracts. The reducing capacity of compounds could serve as an indicator of potential antioxidant property. In the present study, the percentage of scavenging effect on the DPPH radical was concomitantly increased with the increase in the concentration of MESL Fraction A from 5 to $30 \mu \mathrm{g} / \mathrm{ml}$. The percentage of inhibition was existing with 32.88 at $5 \mu \mathrm{g} / \mathrm{ml}$ to 94.28 at $30 \mu \mathrm{g} / \mathrm{ml}^{\left[{ }^{[29]}\right.}$

\section{Total flavonoids content:}

The flavonoids content was determined by aluminum trichloride method using catechin as reference compounds. A volume of $125 \mu \mathrm{L}$ of the extract is added to $75 \mu \mathrm{L}$ of a $5 \% \mathrm{NaNO}_{2}$ solution. The mixture was allowed to stand for $6 \mathrm{~min}$, then $150 \mu \mathrm{L}$ of aluminum trichloride (10\%) was added and incubated for $5 \mathrm{~min}$, followed by the addition of $750 \mu \mathrm{L}$ of $\mathrm{NaOH}(1 \mathrm{M})$. The final volume of the solution was adjusted to $2500 \mu \mathrm{L}$ with distilled water. After 15 min of incubation, the mixture turned to pink and the absorbance was measured at $510 \mathrm{~nm}$. The total content of flavonoids was expressed as g E catechin 100 g-1DM. ${ }^{[30]}$

\section{Scopolamine-induced dementia}

A total of 42 rats were taken, each group contains six rats.

- Group I: Received normal saline

- Group II: Received Scopolamine $5 \mathrm{mg} / \mathrm{kg}$ (i.p.) for 7 days and allowed access to normal food and water

- Group III: Received scopolamine $5 \mathrm{mg} / \mathrm{kg}$ (i.p.). Donepezil $2 \mathrm{mg} / \mathrm{kg}$ for 7 days

- Group IV: Received Scopolamine $5 \mathrm{mg} / \mathrm{kg}$ (i.p.). MESL Fraction A $50 \mathrm{mg} / \mathrm{kg}$ for 7 days

- Group V: Received Scopolamine $5 \mathrm{mg} / \mathrm{kg}$ (i.p.). MESL Fraction A $100 \mathrm{mg} / \mathrm{kg}$ for 7 days

- Group VI: Received Scopolamine $5 \mathrm{mg} / \mathrm{kg}$ (i. p.). MESL crude extract $250 \mathrm{mg} / \mathrm{kg}$ for 7 days

- Group VII: Received Scopolamine $5 \mathrm{mg} / \mathrm{kg}$ (i. p.). MESL crude extract $500 \mathrm{mg} / \mathrm{kg}$ for 7 days.

At the end of the treatment period, mice underwent behavior study for 5 days to evaluate learning and memory status. At experiment termination, mice were sacrificed and brain tissues were collected. Each brain was divided into halves; right and left half hemisphere. One half was fixed and stored in $10 \%$ formaldehyde for histopathological studies. The second half was stored at $-80^{\circ} \mathrm{C}$ for biochemical investigations.

\section{Evaluation parameters Behavioral parameters \\ Escape latency time (s) using Morris water maze}

The Morris water maze is one of the most widely used tasks in behavioral neuroscience for studying the psychological processes and neural mechanisms of spatial learning and memory. The basic task is very simple. Rats are placed in a Morris water maze and required to escape from water onto a hidden platform whose location can normally be identified only using spatial memory. There are no local cues indicating where the platform is located. Conceptually, the task derives from Place cells that are neurons in the hippocampus which identify or represent points in space in an environment. ${ }^{[31]}$

\section{Active avoidance testing using elevated plus maze}

The test uses an elevated, plus-shaped (+) apparatus with two open and two enclosed arms. The behavioral model is based on the general aversion of rodents to open spaces. This aversion leads to the behavior termed thigmotaxis, a preference for remaining in enclosed spaces or close to the edges of a bounded space. In the elevated plus-maze (EPM), this translates into the animals limiting their movement to the enclosed arms.

Anxiety reduction is indicated in the plus-maze by an increase in the proportion of time spent in the open arms (time in open arms/total time in open or closed arms) and an increase in the proportion of entries into the open arms (entries into open arms/total entries into open or closed arms). The total number of arm entries and number of closed-arm entries are sometimes used as measures of general activity. The relationship between the EPM and other tests of exploratory activity (open-field and emergence) has been analyzed.

\section{Neurochemical parameters Estimation of acetylcholinesterase levels}

The activity of AChE has been estimated in hippocampus using acetylthiocholine iodide as a substrate following the colorimetric method. Briefly, the reaction mixture in a final volume of $1.0 \mathrm{ml}$ contained phosphate buffer $(0.1 \mathrm{M}, \mathrm{pH} 7.4)$, post mitochondrial fraction of hippocampus containing around $15-20 \mu$ g protein, acetylthiocholine iodide and 5'dithionitrobenzoic acid (DTNB) $(5 \mathrm{~mm})$. The degradation of acetylthiocholine iodide was measured at $412 \mathrm{~nm}$ and the results are expressed as $\mu$ moles/mg protein. ${ }^{[32]}$

\section{Estimation of reduced glutathione in brain}

Levels of GSH in hippocampus were measured following the standard method. Briefly, $10 \%$ homogenate was deproteinized with an equal volume of trichloroacetic acid (10\%) and allowed to stand at $4^{\circ} \mathrm{C}$ for $1 \mathrm{~h}$. The contents were centrifuged at $3000 \times g$ for $15 \mathrm{~min}$. The supernatant $(0.5 \mathrm{ml})$ was added to $2 \mathrm{ml}$ of Tris- $\mathrm{HCl}$ buffer $(0.4 \mathrm{M}, \mathrm{pH}$ 8.9) containing ethylenediaminetetraacetic acid $(0.02 \mathrm{M}, \mathrm{pH}$ 8.9) followed by the addition of 5, DTNB, $0.01 \mathrm{M}$. The volume was made up to $3 \mathrm{ml}$ by addition of $0.5 \mathrm{ml}$ of distilled water and absorbance of yellow color read on a spectrophotometer at $412 \mathrm{~nm}$, and the results are expressed as $\mu \mathrm{g}$ $\mathrm{GSH} / \mathrm{g}$ tissue. ${ }^{[33]}$

\section{The estimation of superoxide dismutase activity}

The activity of superoxide dismutase (SOD) in hippocampus was measured following the method of Kakkar et al., with slight modifications. The reaction mixture was mixed vigorously with $4.0 \mathrm{ml}$ of $\mathrm{n}$-butanol and the mixture was allowed to stand for 10 min followed by centrifugation for $10 \mathrm{~min}$ at $3000 \times g$ to separate the butanol layer. The color intensity of the chromogen (purple) in butanol layer was measured at $560 \mathrm{~nm}$ against butanol on spectrophotometer. A mixture without enzyme preparations was run in parallel to serve as reagent blank. The activity of SOD is expressed in units $/ \mathrm{min} / \mathrm{mg}$ protein. One unit of the enzyme is the amount required to inhibit the rate of chromogen formation by $50 \%$.

\section{Estimation of catalase activity}

The activity of CAT in hippocampus was assayed following the method of Aebi, using $\mathrm{H}_{2} \mathrm{O}_{2}$ as substrate. Briefly, the reaction mixture in a 
final volume of $1.0 \mathrm{ml}$ contained phosphate buffer $(0.1 \mathrm{mM}, \mathrm{pH} 7.4)$, postmitochondrial fraction of sample $(100 \mu \mathrm{l})$, and $\mathrm{H}_{2} \mathrm{O}_{2}(30 \mathrm{mM})$. The decrease in optical density was measured for $150 \mathrm{~s}$ at $240 \mathrm{~nm}$ using the spectrophotometer. The activity of the enzyme was calculated using the molar extinction coefficient of $43.6 \mathrm{M} / \mathrm{cm}$.

\section{Histopathological studies}

Because of the important role of hippocampus in the memory, its histopathological was investigated. Hence, the removed rat's hippocampal tissues were fixed in $10 \%$ neutral buffered formaldehyde for $24 \mathrm{~h}$, embedded in paraffin and cut into 3-4 $\mu \mathrm{m}$ thick sections by a microtome (Leica SM2000R, Germany). The tissue sections were de-paraffinized in xylene. The slides were stained with Hematoxylin and Eosin according to the procedure of Wilson et al. and viewed under a light microscope (Labomed, USA) for the structure and morphology of cells. Microscopic images obtained by a CCD camera and Digipro software. The cells also were counted in different regions of hippocampus (CA1, CA2, CA3 and DG) by the grade of light microscope. The results are represented as a cell count per $\mathrm{mm}^{2}$ tissue. Furthermore, some of the slides were investigated by Immunohistochemical methods. In these processes, the primary and secondary antibodies were used and Caspase 3 (an apoptotic cytoplasmic protein) was detected as brown color after making complex with DAB (DiaminoBenzidine).

\section{Statistical analysis}

The results were expressed as mean \pm standard error of mean $(n=6)$. Statistical analysis was performed using one way ANOVA followed by Dunnett's comparison test T. $P$ values (expressed) calculated against the Scopolamine group and $P<0.001$ was considered as significant.

\section{RESULTS}

\section{Phytochemical analysis}

Phytochemical studies of Fraction A (Hexane: Ethyl acetate) revealed the Fraction presence of Saponins, phenolics, and flavonoids [Table 2].

\section{Acute oral toxicity study}

Acute oral toxicity was carried out according to OECD guidelines 423 (2001) regulation method.

That MESL was safe at limit dose $2000 \mathrm{mg} / \mathrm{kg}$ [Table 3].

\section{Total flavonoids content of methanolic extract of Sapindus laurifolia Fraction A}

The \% yield of methanolic extract of sapindus laurifolia is $20.4 \mathrm{w} / \mathrm{w}$ and total flavonids content of MESL fraction A is $106.75 \pm 10$ which is compared with standard Catechin [Table 4].

\section{Behavioral parameters \\ Effect of methanolic extract of Sapindus laurifolia extract and fraction A on escape latency using Morris water maze}

Morris water maze task training for 7 days leads to progressive improvement in the ability of rats to explore the hidden platform in the target quadrant. The scopolamine-treated animals exhibited longer escape latencies (time taken to find platform) throughout training days than vehicle-treated controls indicating impairment of memory. The saline-treated control group rapidly learned the location of the platform. MESL Fraction A-treated groups significantly attenuated the effects of scopolamine on escape latency (EL) as did donepezil-treated groups [Figure 1].
Table 2: Identification of phytochemical constituents

\begin{tabular}{lcccccc}
$\begin{array}{lcccc}\text { Phytochemical } \\
\text { tests }\end{array}$ & $\begin{array}{c}\text { H: EA } \\
(95: 5)\end{array}$ & $\begin{array}{c}\text { H: EA } \\
(7030)\end{array}$ & $\begin{array}{c}\text { H: EA } \\
(50: 50)\end{array}$ & $\begin{array}{c}\text { EA } \\
\text { only }\end{array}$ & $\begin{array}{c}\text { H } \\
\text { only }\end{array}$ & $\begin{array}{c}\text { M } \\
\text { only }\end{array}$ \\
\hline Alkaloids & - & - & - & - & - & - \\
Glycosides & - & - & - & - & - & - \\
Tannins & - & - & - & - & - & - \\
Phenols & ++ & - & - & - & - & - \\
Flavonoids & ++ & ++ & - & - & - & - \\
Saponins & ++ & - & - & - & - & - \\
Steroids & ++ & ++ & & & & \\
\hline
\end{tabular}

+: Presence; -: Absence; H: Hexane; EA: Ethyl acetate; M: Methanol

Table 3: Acute oral toxicity study of Fraction A

\begin{tabular}{lccc}
\hline Groups & Dose $(\mathbf{m g} \mathbf{k g})$ & Behavioral changes & Mortality rate \\
\hline Group I & 5 & Nil & Nil \\
Group II & 50 & Nil & Nil \\
Group III & 300 & Nil & Nil \\
Group IV & 2000 & Nil & Nil \\
\hline
\end{tabular}

Table 4: Total flavonoids content of methanolic extract of Sapindus laurifolia Fraction A

\begin{tabular}{lcc}
\hline Sample & Percentage yield (W/W) & Total flavanoids content \\
\hline Fraction A & 20.4 & $106.75 \pm 10$ \\
\hline
\end{tabular}

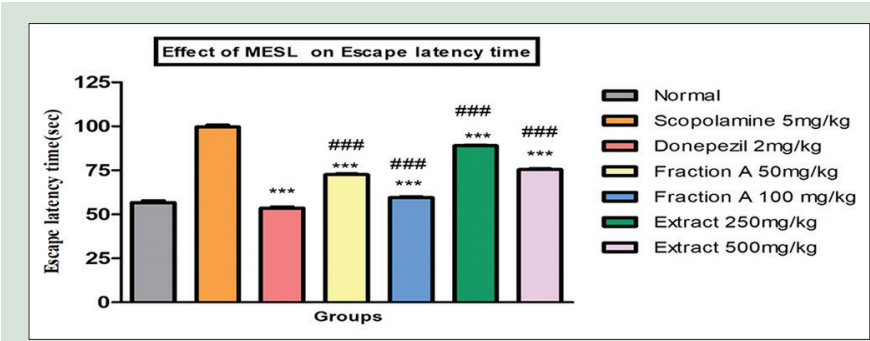

Figure 1: Effect of methanolic extract of Sapindus laurifolia extract and fraction A on escape latency. All values are expressed in mean \pm standard error of mean. Scopolamine-treated group animals show increase in escape latency time when compared to normal control group of animals, whereas donepezil-treated animals decrease in escape latency time (***P $<0.001)$ when compared to scopolamine-treated animals and methanolic extract of Sapindus laurifolia fraction A $(100 \mathrm{mg} / \mathrm{kg})$ and methanolic extract of Sapindus laurifolia extract $(500 \mathrm{mg} / \mathrm{kg})$ treated animals significantly decrease in escape latency time $(\# \# P<0.001)$ compared to scopolamine-treated animals

\section{Effect of methanolic extract of Sapindus laurifolia extract and fraction $A$ on passive avoidance}

Exposure of rats to scopolamine caused a significant decrease in Escape latency time (ELT) of the retention trials as compared to the acquisition trial indicating decrease in the learning and memory activity of rats in comparison to controls. The TLT in rats in the control and in simultaneous treatment with donepezil and scopolamine group were significantly increased in the retention trial as compared to acquisition trial. Furthermore, simultaneous treatment of MESL Fraction A with scopolamine separately in rats caused a significant increase in the in the retention trial as compared to acquisition trial. The treatment of HEMBS and scopolamine in rats caused a significant increase in the retention trial as compared to the acquisition trial indicating improved learning and memory in rats [Figure 2]. 


\section{Neurochemical parameters}

Effect of methanolic extract of Sapindus laurifolia extract and

\section{Fraction $A$ on acetylcholinesterase levels}

Scopolamine-treated group animals show increase in acetylcholinesterase levels when compared to normal control group of animals, whereas donepezil-treated animals decrease in acetylcholinesterase levels $\left({ }^{* *} P<0.001\right)$ when compared to scopolamine-treated animals and methanolic extract of Sapindus laurifolia Fraction A-treated animals significantly decrease in acetylcholinesterase levels $\left({ }^{\# \#} P<0.001\right)$ compared to scopolaminetreated animals, whereas methanolic extract of Sapindus laurifolia extract-treated group animals does not show significant effect when compared to methanolic extract of Sapindus laurifolia Fraction A-treated group. Based on these results MESL fraction A significantly reduces the acetyl cholinesterase levels [Figure 3].

\section{Effect of methanolic extract of Sapindus laurifolia extract and Fraction $A$ on catalase}

Scopolamine-treated group animals show decrease in catalase levels when compared to normal control group of animals, whereas donepeziltreated animals decrease in catalase levels $\left({ }^{* *} P<0.001\right)$ when compared to scopolamine-treated animals and methanolic extract of Sapindus laurifolia fraction A $(100 \mathrm{mg} / \mathrm{kg})$ and methanolic extract of Sapindus laurifolia extract $(500 \mathrm{mg} / \mathrm{kg})$ treated animals significantly increase in catalase levels $\left.{ }^{\# \# \# P} P<0.001\right)$ compared to scopolamine-treated animals. Based on these results MESL fraction A significantly increases the catalase levels [Figure 4].

\section{Effect of methanolic extract of Sapindus laurifolia extract and Fraction $A$ on glutathione}

Scopolamine-treated group animals show decrease in glutathione levels

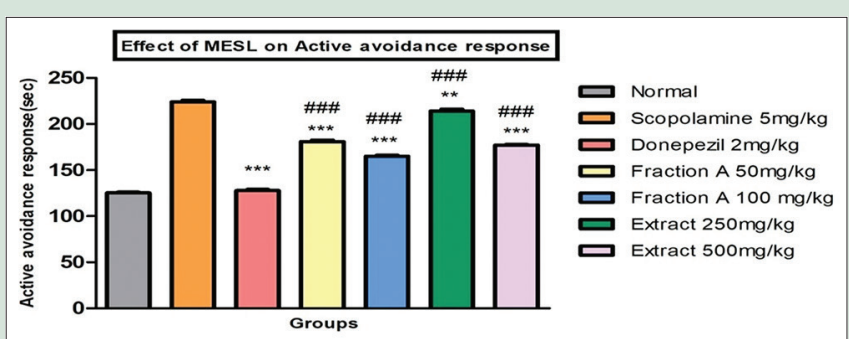

Figure 2: Effect of methanolic extract of Sapindus laurifolia extract and fraction A on passive avoidance response. All values are expressed in mean \pm standard error of mean. Scopolamine-treated group animals show increase in active avoidance response when compared to normal control group of animals, whereas donepezil-treated animals decrease in escape latency time ( $\left.{ }^{* *} P<0.001\right)$ when compared to scopolaminetreated animals and methanolic extract of Sapindus laurifolia fraction A $(100 \mathrm{mg} / \mathrm{kg})$ and methanolic extract of Sapindus laurifolia extract $(500 \mathrm{mg} / \mathrm{kg})$ treated animals significantly decrease in active avoidance response ( $\left.{ }^{\# \# P}<0.001\right)$ compared to scopolamine-treated animals when compared to normal control group of animals, whereas donepeziltreated animals increase in glutathione levels $\left({ }^{\star * *} P<0.001\right)$ when compared to scopolamine-treated animals and methanolic extract of Sapindus laurifolia fraction A $(100 \mathrm{mg} / \mathrm{kg})$ treated animals significantly increase in glutathione levels ( ${ }^{\# \# P} P<0.001$ ) compared to scopolaminetreated animals, whereas methanolic extract of Sapindus laurifolia extract-treated group animals does not show significant effect when compared to Fraction A group. Based on these results MESL fraction A significantly increases the glutathione levels. High levels of glutathione decreases the oxidative stress induced dementia [Figure 5].

\section{Effect of methanolic extract of Sapindus laurifolia extract and Fraction A on SOD levels}

Scopolamine-treated group animals show decrease in superoxide dismutase levels when compared to normal control group of animals, whereas donepezil-treated animals increase in superoxide dismutase levels $\left({ }^{* *} P<0.001\right)$ when compared to scopolamine-treated animals and methanolic extract of Sapindus laurifolia fraction A $(100 \mathrm{mg} /$ $\mathrm{kg}$ ) and methanolic extract of Sapindus laurifolia extract $(500 \mathrm{mg} / \mathrm{kg}$ ) treated animals significantly increase in superoxide dismutase levels (\#\# $P<0.001)$ compared to scopolamine-treated animals. Based on these results MESL fraction A significantly increases the SOD levels [Figure 6].

\section{Effect of methanolic extract of Sapindus laurifolia extract and Fraction A on lipid peroxidase levels}

Scopolamine-treated group animals increase in lipid peroxidase levels when compared to normal control group of animals, whereas donepeziltreated animals decrease in lipid peroxidase levels $\left({ }^{* * *} P<0.001\right)$ when

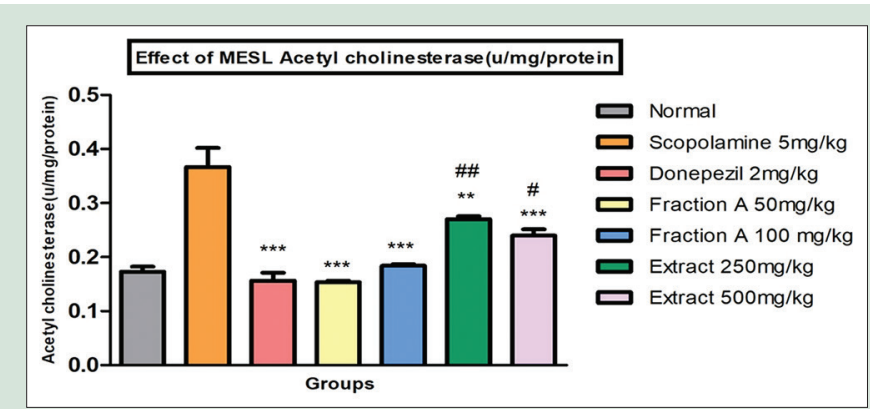

Figure 3: Effect of methanolic extract of Sapindus laurifolia extract and fraction A on acetylcholinesterase. All values are expressed in mean \pm standard error of mean. Scopolamine-treated group animals show increase in acetylcholinesterase levels when compared to normal control group of animals, whereas donepezil-treated animals decrease in acetylcholinesterase levels $\left({ }^{* * *} P<0.001\right)$ when compared to scopolaminetreated animals and methanolic extract of Sapindus laurifolia Fraction A-treated animals significantly decrease in acetylcholinesterase levels $\left({ }^{\# \# P} P\right.$ 0.001) compared to scopolamine-treated animals, whereas methanolic extract of Sapindus laurifolia extract-treated group animals does not show significant effect when compared to methanolic extract of Sapindus laurifolia Fraction A-treated group

Table 5: Free radical scavenging activity (2,2-diphenyl-1-picrylhydrazyl) of Fraction A

\begin{tabular}{|c|c|c|c|c|}
\hline $\begin{array}{l}\text { Concentration } \\
\text { (ug/ml) }\end{array}$ & $\begin{array}{c}\text { Absorbance } \\
\text { (ascorbic acid) }\end{array}$ & $\begin{array}{c}\text { Absorbance of } \\
\text { (Fraction A) }\end{array}$ & $\begin{array}{c}\text { Percentage inhibition } \\
\text { of ascorbic acid }\end{array}$ & $\begin{array}{c}\text { Percentage inhibition } \\
\text { (Fraction } \mathrm{A})\end{array}$ \\
\hline 50 & 0.2380 & 0.3546 & 46.57 & $32.88 \pm 0.33$ \\
\hline 100 & 0.1719 & 0.3956 & 55.64 & $56.54 \pm 0.36$ \\
\hline 150 & 0.0469 & 0.4629 & 63.82 & $88.91 \pm 0.53$ \\
\hline 200 & 0.0415 & 0.5739 & 65.94 & $92.00 \pm 0.63$ \\
\hline 250 & 0.0410 & 0.5956 & 65.86 & $93.11 \pm 0.55$ \\
\hline $\mathrm{IC}_{50}$ value & & & 65 & 15.54 \\
\hline
\end{tabular}

$\mathrm{IC}_{50}:$ Half maximal inhibitory concentration 
compared to scopolamine-treated animals and methanolic extract of Sapindus laurifolia fraction A $(100 \mathrm{mg} / \mathrm{kg})$ and methanolic extract of Sapindus laurifolia extract-treated animals significantly decreases in lipid peroxidase levels ( ${ }^{\# \# ~} P<0.001$ ) compared to scopolamine-treated animals. Based on the results MESL fraction A significantly decreases the peroxidase levels [Figure 7].

\section{Histopathology of rat brain (hippocampus)}

Histopathological studies of hippocampus revels, scopolaminetreated animal brain show congestion of blood capillaries, formation of perivascular edema and beta amyloidal plaques, where as donepezil and MESL treated animals brains show decreased the formation of perivascular edema and beta amyloidal plaques, so it indicates the MESL show neuroprotective action in scopolamine induced dementia [Figure 8].

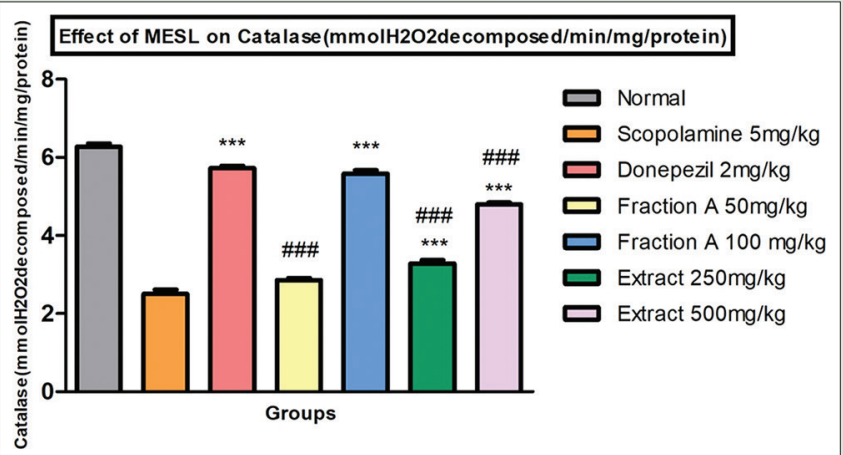

Figure 4: Effect of methanolic extract of Sapindus laurifolia extract and fraction A on catalase. All values are expressed in mean \pm standard error of mean. Scopolamine-treated group animals show decrease in catalase levels when compared to normal control group of animals, whereas donepezil-treated animals decrease in catalase levels $\left({ }^{* * *} P<0.001\right)$ when compared to scopolamine-treated animals and methanolic extract of Sapindus laurifolia fraction A (100 mg/kg) and methanolic extract of Sapindus laurifolia extract $(500 \mathrm{mg} / \mathrm{kg})$ treated animals significantly increase in catalase levels $(\# \# P<0.001)$ compared to scopolamine-treated animals

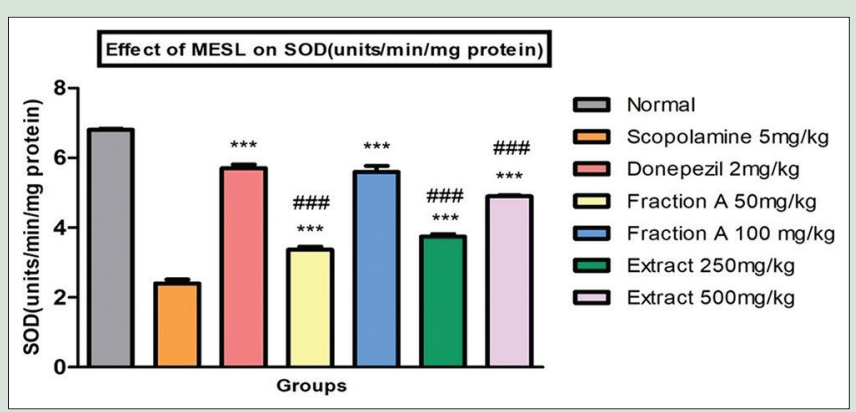

Figure 6: Effect of methanolic extract of Sapindus laurifolia extract and fraction A on superoxide dismutase levels. All values are expressed in mean \pm standard error of mean. Scopolamine-treated group animals show decrease in superoxide dismutase levels when compared to normal control group of animals, whereas donepezil-treated animals increase in superoxide dismutase levels $\left({ }^{* *} P<0.001\right)$ when compared to scopolamine-treated animals and methanolic extract of Sapindus laurifolia fraction A $(100 \mathrm{mg} / \mathrm{kg})$ and methanolic extract of Sapindus laurifolia extract $(500 \mathrm{mg} / \mathrm{kg})$ treated animals significantly increase in superoxide dismutase levels (\#\#P<0.001) compared to scopolaminetreated animals

\section{DISCUSSION}

Medicinal plants are storehouse of phytochemicals for the treatment of countless major and minor disease. In the treatment of the neurodegenerative, particularly $\mathrm{AD}$, the phytoconstituents of medicinal plants play a crucial role as stated earlier. Flavonoids are a group of polyphenolic compounds, which exhibit several biological effects such as anti-inflammatory, Hepatoprotective, antiulcer, antiallergic, antiviral, and anticancer activities. They are capable of effectively scavenging the reactive $\mathrm{O}_{2}$ species because of their phenolic hydroxyl groups and so they are potent antioxidants also, the total flavonoids content of MESL Fraction A was determined to be $106.75 \mu \mathrm{g} / 2 \mathrm{mg}$, respectively, in terms of $\mathrm{g}$ E catechin.100 g-1DM [Table 4].

To find out oxidative stress at various levels, the defensive antioxidant enzymes in rat brain were measured. DPPH scavenging activity of

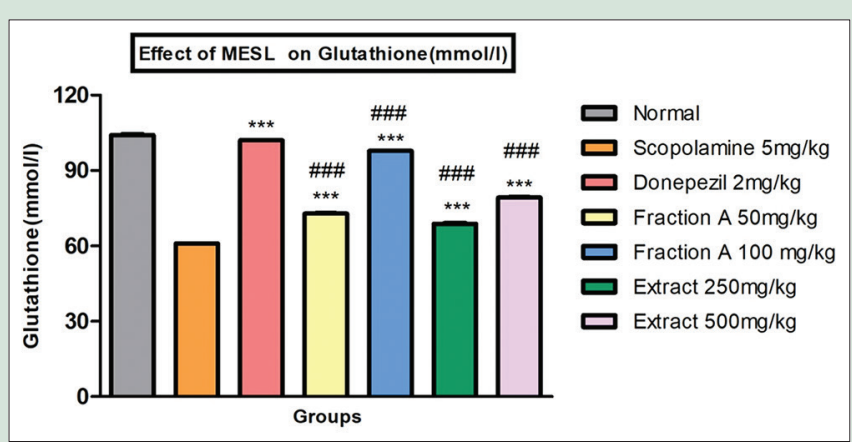

Figure 5: Effect of methanolic extract of Sapindus laurifolia extract and fraction A on glutathione. All values are expressed in mean \pm standard error of mean. Scopolamine-treated group animals show decrease in glutathione levels when compared to normal control group of animals, whereas donepezil-treated animals increase in glutathione levels ${ }^{* * *} P<$ 0.001) when compared to scopolamine-treated animals and methanolic extract of Sapindus laurifolia fraction A $(100 \mathrm{mg} / \mathrm{kg})$ treated animals significantly increase in glutathione levels $\left({ }^{\# \# P} P<0.001\right)$ compared to scopolamine-treated animals, whereas methanolic extract of Sapindus laurifolia extract-treated group animals does not show significant effect when compared to Fraction A group

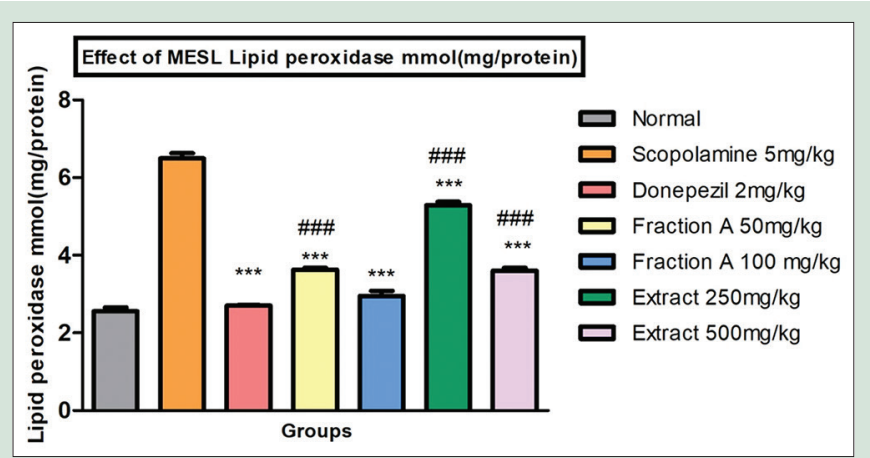

Figure 7: Effect of methanolic extract of Sapindus laurifolia extract and fraction A on lipid peroxidase levels. All values are expressed in mean \pm standard error of mean. Scopolamine-treated group animals increase in lipid peroxidase levels when compared to normal control group of animals, whereas donepezil-treated animals decrease in lipid peroxidase levels (*** $P<0.001$ ) when compared to scopolamine-treated animals and methanolic extract of Sapindus laurifolia fraction A (100 mg/kg) and methanolic extract of Sapindus laurifolia extract-treated animals significantly decreases in lipid peroxidase levels $(\# \# P<0.001)$ compared to scopolamine-treated animals 


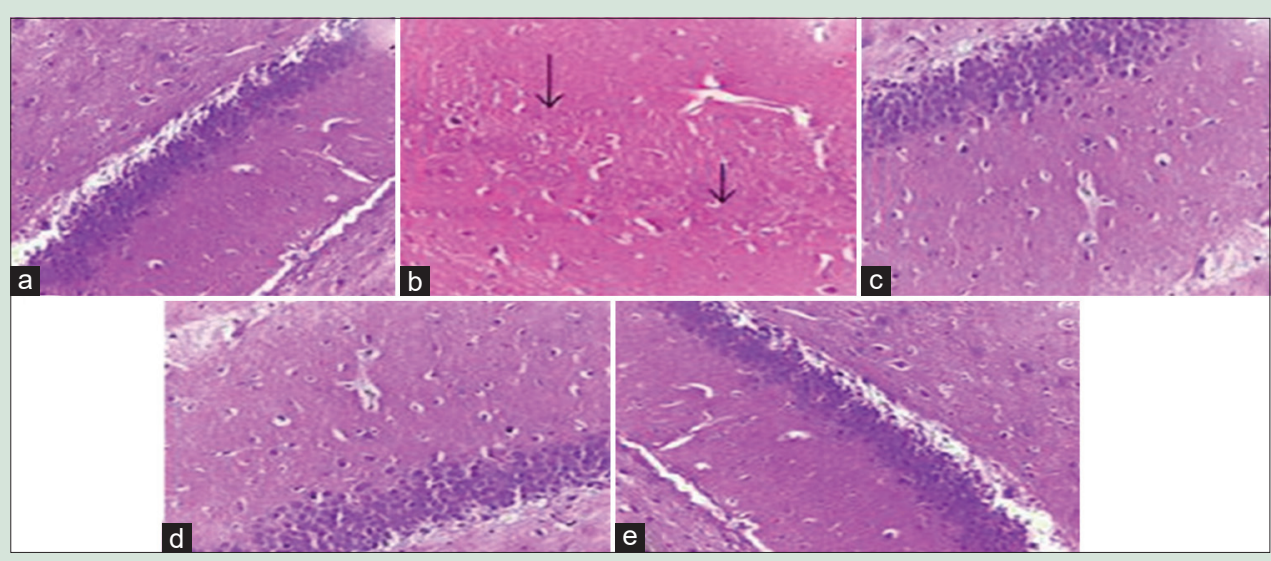

Figure 8: Histopathological studies of rat brain: (a) Normal control rat showing the normal histological structure of Hippocampus. (b) Scopolamine induced dementia rat showing severe congestion of blood capillaries with perivascular edema (Scars) together with edema and amyloid plaques in hippocampus. (c) When compared with control, fraction-treated rat show less formation of amyloid plaques in hippocampus region of the brain. (d) Scopolamine induce dementia rat treated with donepezil showing diffuse gliosis and shrinkage of nuclei in pyramidal cell in hippocampus. (e) When compared with control, fraction high-dose treated rat show less formation of amyloid plaques in hippocampus region of the brain. (a) Normal control. (b) Diseased control. (c) Methanolic extract of Sapindus laurifolia (50 mg) + scopolamine. (d) Donepezil $2 \mathrm{mg}+$ scopolamine. (e) Methanolic extract of Sapindus laurifolia Fraction A $(100 \mathrm{mg})+$ scopolamine

fraction $\mathrm{A}\left(\mathrm{IC}_{50}\right.$ 15.54) showed the $\mathrm{IC}_{50}$ value close to the ascorbic acid $\left(\mathrm{IC}_{50} 65\right)$ [Table 5].

The scopolamine-induced animal model of dementia and oxidative stress is widely used as a primary screening test for the determination of anti-Alzheimer effect of unknown plants or drugs in this study, MESL administration for $7^{\text {th }}$ day showed significant neuroprotective effect by improving various types of memory, learning, antioxidant enzymes, and anti-AchE activity in rats. This is the first study showing neuroprotective activity of MESL Fraction A in rats by using various behavioral and biochemical studies. The Morris water maze model is widely used to assess the effects of drugs on learning and memory. In this model, a decrease in EL and an increase in time spent in the target quadrant (TSTQ) indicate improvement of learning and memory. The MESL Fraction A showed a significant decrease in EL and a significant increase in TSTQ in scopolamine mice.

Spatial long-term memory is evaluated by EPM test in which the measured parameters were ITL and RTL. The time spent by the rat to move from the open arm to the closed arm in this test was recorded as ITL. After $24 \mathrm{~h}$ of long-term memory, the retention of the learned task was studied as RTL. In this test, a decrease in RTL on $7^{\text {th }}$ day after the ILT on $6^{\text {th }}$ day respectively indicated improvement of spatial long-term memory of rats as compared to disease control and control group.

The MESL Fraction A also showed positive effects on rat brain. In our study, IP injection of scopolamine increased the levels of LPO it indicates the increased levels of ROS because it catalyzed by ROS. LPO may become very harmful due to the fact that a single-free radical can cause damage to a number of polyunsaturated fatty acid (PUFA) molecules. PUFA can produce more neurotoxic molecules such as malondialdehyde and acrolein. The brain is specifically vulnerable to oxidative stress and LPO.

The results of our study show that after IP injection of scopolamine, the activity of SOD was decreased in the brain which could be a result of the inactivation of SOD by $\mathrm{H}_{2} \mathrm{O}_{2}$. This result intimated a compensatory response to oxidative stress due to an increase in the synthesis of endogenous $\mathrm{H}_{2} \mathrm{O}_{2}$. Therefore, the elevation in the level of SOD in MESL Fraction A-treated animals foretells that MESL Fraction A may possess free radical scavenging potential, which could be beneficial against the pathological alterations produced by the presence of $\mathrm{O}_{2}$ and $\mathrm{OH}$.
One of the most important antioxidants in mammalian cells is glutathione (GSH). This nonprotein thiol antioxidant is the major intracellular redox buffer. GSH-dependent detoxification involves GSH-Px, which has a pivotal role in the elimination of hydrogen and organic peroxides and leads to the formation of oxidized glutathione but is reduced back to its thiol form (GSH) by glutathione reductase (GSHr), leading to the consumption of Nicotinamide adenine dinucleotide phosphate (NADPH), which is chiefly formed by pentose phosphate pathway. GSH is involved in xenobiotic conjugation with the help of different glutathione S-transferase isoenzymes. The inhibition of GSH synthesis causes an increase in $A \beta$ - mediated cell death and intracellular $A \beta$ accumulation. Various clinical trials have reported strong evidence that impairment in memory observed in rodents is linked with the altered levels of GSH in the brain and with the activities of the antioxidant enzyme. Increase of brain oxidative status of amnesic rodents was similar as observed in the clinical pathology of AD patients. Scopolamine-mediated increase in LPO and decreases in glutathione (GSH), a natural antioxidant, are one of the principal causes of neurodegeneration.

In our study, scopolamine administration caused a significant decrease in the activity of GSH-Px, glutathione reductase (GSHr), catalase, and SOD in Alzheimer group Rats. The administration of methanolic extract of S. laurifolia kernel restored the levels of enzymes in Scopolamine injected rats to similar levels as seen in normal control rats. Hence, we can conclude that the extract possesses the neuroprotective activity in rats [Figures 3-7]. The neuroprotective activity of $S$. laurifolia Fraction A can be attributed to the presence of flavonoids and polyphenolic compounds in the leaves of the plant. Therefore, in future, we intend to conduct studies on isolated compounds of the leaves of this plant.

\section{CONCLUSION}

The present study clearly demonstrates that MESL Fraction A (50 and $100 \mathrm{mg} / \mathrm{kg}$ ) significantly attenuates scopolamine-induced dementia by improving the learning, memory, antioxidant potentiality, and anti-AchE activity. Therefore, this fraction can be a potential novel therapeutic strategy for controlling neurodegenerative dementia, especially AD. Yet, advance studies are needed to characterize the active compound(s) and expose the possible mechanism of action 


\section{Acknowledgements}

The authors are thankful to the Management of Shri Vishnu College of Pharmacy, Bhimavaram and Management of Chebrolu Hanumaiah Institute of Pharmaceutical Sciences, Guntur.

\section{Financial support and sponsorship}

This study was supported by Research center and Self.

\section{Conflicts of interest}

There are no conflicts of interest.

\section{REFERENCES}

1. Sternberg RJ Pretz JE. Cognition and Intelligence: Identifying the Mechanisms of the Mind. New York: Cambridge University Press; 2005.

2. Selnes OA, Vinters HV. Vascular cognitive impairment. Nat Clin Pract Neurol 2006;2:538-47.

3. Sosa AL, Albanese E, Stephan BC, Dewey M, Acosta D, Ferri CP, et al. Prevalence, distribution, and impact of mild cognitive impairment in Latin America, China, and India: A 10/66 population-based study. PLoS Med 2012;9:e1001170.

4. Querfurth HW, LaFerla FM. Alzheimer's disease. N Engl J Med 2010;362:329-44

5. Wimo A, Winblad B, Aguero-Torres $H$, von Strauss E. The magnitude of dementia occurrence in the world. Alzheimer Dis Assoc Disord 2003;17:63-7.

6. Asaduzzaman M, Uddin MJ, Kader MA, Alam AH, Rahman AA, Rashid M, et al. In vitro acetylcholinesterase inhibitory activity and the antioxidant properties of Aegle marmelos leaf extract: implications for the treatment of Alzheimer's disease. Psychogeriatrics 2014;14:1-10.

7. Chang YT, Chang WN, Tsai NW, Huang CC, Kung CT, Su YJ, et al. The roles of biomarkers of oxidative stress and antioxidant in Alzheimer's disease: A systematic review. Biomed Res Int 2014;2014:182-303.

8. Oh JH, Choi BJ, Chang MS Park SK. Nelum bonucifera semen extract improves memory in rats with scopolamine-induced amnesia through the induction of choline acety Itransferase expression. Neurosci Lett 2009;461:41-4.

9. Fan Y, Hu J, Li J, Yang Z, Xin X, Wang J, et al. Effect of acidic oligosaccharide sugar chain on scopolamine-induced memory impairment in rats and its related mechanisms. Neurosci Lett 2005;374:222-6.

10. El-Sherbiny DA, Khalifa AE, Attia AS, Eldenshary Eel-D. Hypericum perforatum extract demonstrates antioxidant properties against elevated rat brain oxidative status induced by amnestic dose of scopolamine. Pharmacol Biochem Behav 2003;76:525-33

11. Jeong EJ, Lee KY, Kim SH, Sung SH, Kim YC. Cognitive-enhancing and antioxidant activities of iridoid glycosides from Scrophularia buergeriana in scopolamine-treated mice. Eur J Pharmacol 2008;588:78-84..

12. Sultana $R$, Butterfield DA. Role of oxidative stress in the progression of Alzheimer 's disease. J Alzheimers Dis 2010;19:341-53

13. Hossain MS, Asaduzzaman M, Uddin MS, Noor MA, Rahman MA Munira MS, et al. Investigation of the in vitro antioxidant and cytotoxic activities of Xanthosoma sagittifolium leaf. Adv Alzheimer Dis 2016;5:5-3300.

14. Kuhla B, Haase C, Flach K, Lüth HJ, Arendt T, Münch G. Effect of pseudophosphorylation and cross-linking by lipid peroxidation and advanced glycation end product precursors on tau aggregation and filament formation. $J$ Biol Chem 2007;282:6984-91.

15. Zimmerman G, Soreq H. Termination and beyond: Acetylcholinesterase as a modulator of synaptic transmission. Cell Tissue Res 2006;326:655-69.

16. Lane RM, Potkin SG, Enz A. Targeting acetylcholinesterase and butyrylcholinesterase in dementia. Int J Neuropsychopharmacol 2006;9:101-24.

17. Zawia NH, Lahiri DK, Cardozo-Pelaez F. Epigenetics, oxidative stress, and Alzheimer disease. Free Radic Biol Med 2009;46:1241-9.

18. Jayakumar T, Thomas PA, Geraldine P. Protective effect of an extract of the oyster mushroom, Pleurotus ostreatus, on antioxidants of major organs of aged rats. Exp Gerontol 2007;42:183-91.

19. Gilgun-SherkiY, MelamedE, Offen D. Oxidative stress induced-neurodegenerative diseases: The need for antioxidants that penetrate the blood brain barrier. Neuropharmacology 2001;40:959-75.

20. Uttara B, Singh AV, Zamboni P, Mahajan RT. Oxidative stress and neurodegenerative diseases: A review of upstream and downstream antioxidant therapeutic options. Curr Neuropharmacol 2009;7:65-74

21. Sharma J, Chawla R, Kumar R, Sharma A, Sharma RK, Arora R. Camellia sinensis as a safe neuroprotective radiation counter measure agent. Int J Pharm Sci Invent 2013;2:26-33.

22. Duraipandiyan $V$, Ayyanar M, Ignacimuthu S. Antimicrobial activity of some ethnomedicinal plants used by Paliyar tribe from Tamil Nadu, India. BMC Complement Altern Med 2006;6:35.

23. Jamison DT, Breman JG, Meashametal AR. Complementary and alternative medicine: Traditional medicine. In: Disease Control Priorities in Developing Countries. Washington, DC: World Bank; 2014

24. Oken BS, Storzbach DM, Kaye JA. The efficacy of Ginkgo biloba on cognitive function in Alzheimer disease. Arch Neurol 1998;55:1409-15.

25. Ghazouani N, Abderrabba M, Bouajila J. In-vitro evaluation of pharmaceutical activities of Teucriumram osissimum. J Nat Prod 2015;8:106-12.

26. Mathew M, Subramanian S. In vitro evaluation of anti-Alzheimer effects of dry ginger (Zingiber officinale Roscoe) extract. Indian J Exp Biol 2014;52:606-12.

27. Bhuiyan MA, Hoque MZ, Hossain SJ. Free Radical Scavenging activities of Zizyphus mauritiana. World J Agr 2009;5:318-22

28. Kwon SH, Lee HK, Kim JA, Hong SI, Kim HC, Jo TH, et al. Neuroprotective effects of chlorogenic acid on scopolamine-induced amnesia via anti-acetylcholinesterase and anti-oxidative activities in mice. Eur J Pharmacol 2010;649:210-7.

29. Tota S, Nath C, Najmi AK, Shukla R, Hanif K. Inhibition of central angiotensin converting enzyme ameliorates scopolamine induced memory impairment in mice: Role of cholinergic neurotransmission, cerebral blood flow and brain energy metabolism. Behav Brain Res 2012;232:66-76.

30. Sumanth M, Sowmya H, Nagaraj SV, Narasimharaju K. Efficacy of donepezil and galantamine in retrograde amnesia. AJPCR 2010;3:23-5.

31. Ellman GL, Courtney KD, Andres V Jr., Feather-Stone RM. A new and rapid colorimetric determination of acetylcholinesterase activity. Biochem Pharmacol $1961 ; 7: 88-95$

32. Hasan M, Haider SS. Acetyl-homocysteine thiolactone protects against some neurotoxic effects of thallium. Neurotoxicology 1989;10:257-61.

33. Kakkar P, Das B, Viswanathan PN. A modified spectrophotometric assay of superoxide dismutase. Indian J Biochem Biophys 1984;21:130-2. 